\title{
Stereotactic radiosurgery as a primary treatment modality for pineal parenchymal tumors
}

\author{
S. Ahn', J.S. Park ${ }^{1}$ J.H. Song ${ }^{2}$, Y.K. Hong1, S.S. Jeun ${ }^{*}$ \\ ${ }^{1}$ Department of Neurosurgery, Seoul St. Mary's Hospital, College of Medicine, The Catholic University of Korea, \\ Seoul, Korea \\ 2Department of Radiation Oncology, Seoul St. Mary's Hospital, College of Medicine, The Catholic University of \\ Korea, Seoul, Korea
}

\section{- Original article}

\section{*Corresponding authors:}

Sin-Soo Jeun, M.D., Ph.D., E-mail:

ssjeun@catholic.ac.kr

Revised: January 2020

Accepted: February 2020

Int. J. Radiat. Res., October 2020; 18(4): $785-790$

DOI: 10.18869 /acadpub.ijrr.18.4.785

\begin{abstract}
Background: Pineal parenchymal tumor (PPT) is extremely rare and histologically heterogeneous, and the optimal treatment strategy is controversial. This study examined clinical outcomes of patients with PPT treated with cyberknife radiosurgery (CKRS) as a primary treatment modality after tumor biopsy. Materials and Methods: Between 2009 and 2018, there were six patients diagnosed with PPT who were treated with single or hypofractionated cyberknife radiosurgery (CKRS) after undergoing endoscopic third ventriculostomy (ETV) and tumor biopsy. Two tumors were pineocytoma (PC) and four were pineal parenchymal tumor of intermediate differentiation (PPTID). The marginal dose was $18 \mathrm{~Gy}$, and the median target volume was 3.05 (range 1.4-7.4) cc. The median follow-up period was 55 (range 18 -141) months. Results: Five patients were alive, and all of their tumors were locally controlled during follow-up periods. Two tumors disappeared completely, two other tumors partially regressed, and another tumor showed no interval change after CKRS. One patient with PPTID showed local progression 14 months after CKRS and died 21 months after CKRS. There were no acute or late complications related to CKRS. Conclusion: This study suggested that CKRS can be used as a primary treatment modality after ETV and tumor biopsy for PPT with favorable clinical outcomes. However, alternative treatment modalities need to be considered for higher risk groups of patients with PPTID.
\end{abstract}

Keywords: Pineal parenchymal tumor, stereotactic radiosurgery, cyberknife, pineocytoma, pineal parenchymal tumor of intermediate differentiation.

\section{INTRODUCTION}

Pineal parenchymal tumor (PPT) is extremely rare and accounts for 14 to $27 \%$ of all pineal region tumors in adults $(1,2)$. Histologically diverse lesions, they are classified as pineocytoma (PC) (grade I), pineal parenchymal tumor of intermediate differentiation (PPTID) (grade II or III), papillary tumor of the pineal region (PTPR) (grade II or III), and pineoblastoma (grade IV) based on the 2016 World Health Organization (WHO) classification of tumors of the central nervous system (CNS) ${ }^{(3)}$. While multimodal treatments including surgical resection, fractionated radiotherapy, stereotactic radiosurgery, and chemotherapy can be considered for these patients, the optimal treatment strategy for each tumor has not been established due to limited case series (4-9).

Gross total surgical resection (GTR) has been suggested to have the best prognosis $(4,6,10)$; however, GTR is not always achieved. In some studies, the GTR rate was only $50 \%$, and perioperative mortality and morbidity were $0-11 \%$ and $19-28 \%$, respectively $(4,8,9,11)$. Considering surgical risks associated with the unique and deep anatomy of the pineal region and the relatively benign prognosis of PPT 
(except for pineoblastoma), there is question of benefit in debulking surgery for treatment of PPT $(8,12)$.

Stereotactic radiosurgery (SRS) has recently been suggested as an effective treatment modality for PPT (5,11-15). The role of SRS as a primary treatment modality without debulking surgery and/or chemotherapy in management of PPT is still unclear, especially for PPTID, which is relatively more aggressive than PC $(4,13)$. In this study, we reviewed clinical outcomes in patients with PPT treated with cyberknife radiosurgery (CKRS) alone after tumor biopsy.

\section{MATERIALS AND METHODS}

\section{Patients' selection}

This study was approved by the institutional review board at our institution (\#KC19RESI0171, 02-Apr-2019). Due to the retrospective manner of this study, the need for informed consent for data collection was waived. The electronic medical records of 10 patients diagnosed with PPT at our hospital between 2009 and 2016 were retrospectively reviewed. The following inclusion criteria were used: 1) newly diagnosed PPT, 2) pathology obtained and confirmed by craniotomy or endoscopic biopsy, and 3) CKRS performed at our hospital.

\section{Clinical characteristics and treatment protocols}

Characteristics of the eligible patients, including sex, age at diagnosis, symptoms, and radiologic findings on magnetic resonance imaging (MRI), extent of surgery, pathologic findings, radiosurgery profile, and adjuvant chemotherapy were reviewed. All MRI findings included gadolin ium-enhanced T1 weighted images, T2 weighted images, and fluidattenuated inversion recovery (FLAIR) images. All eligible patients underwent endoscopic third ventriculostomy (ETV) and tumor biopsy. Hydrocephalus symptoms resolved within a few days after surgery in all patients, and there were no complications during the perioperative period. Detailed pathologic findings and diagnosis, including nuclear atypia, mitosis, cellular pleomorphism, necrosis, endovascular proliferation, and Ki-67 index, were reviewed and described by a board-certified pathologist at our hospital. MRI evaluation was performed six months after stereotactic radiosurgery, and then patients attended annual follow-up visits for five years if no progression was detected. Treatment response was divided into four categories: complete response (CR), partial response (PR) (more than $50 \%$ reduction in size), progressive disease (PD) (more than 25\% increase in size), and stable disease (SD). The primary endpoint in this study was local tumor control (LTC), which was defined as time interval (months) from initial surgery to recurrence detected radiologically. We also investigated overall survival (OS), and dates of death were obtained from the Korea Central Cancer Registry database. Patients were determined to be alive or dead on January 1, 2019. All treatments were discussed and approved by our multidisciplinary team for brain tumors, including a neurosurgeon, radiation oncologist, medical oncologist, and pathologist.

\section{Radiosurgical treatment}

SRS was performed using the Cyberknife (Accuray Inc., Sunnyvale, CA, USA), which is a frameless robotic system that delivers 6-MV photons without a flattening filter. All patients underwent hypofractionated stereotactic radiosurgery administered as accumulated dose of $18 \mathrm{~Gy}$. Due to its very close location to the brain stem, the dose to the brain stem was taken into consideration when planning. We tried to limit the maximal dose of 23.1Gy (7.7 Gy per fraction) for the brain stem in accordance with the recent guidelines of stereotactic body radiation therapy for the brainstem published by the American Association of Physicists in Medicine in 2010 (16).

\section{RESULTS}

Six patients with PPT who were treated with CKRS as a primary treatment modality within three months after ETV and tumor biopsy were included. Two of them were diagnosed with PC,

Int. J. Radiat. Res., Vol. 18 No. 4, October 2020 
while four were diagnosed with PPTID. The mean age was 41.5 (range 8-58) years, and the mean follow-up period was 55 (range 18 -141) months. Initial presenting symptoms resolved immediately after surgery, and there were no perioperative complications.

One of the two patients diagnosed with pineocytoma (Case No. 3) showed partial response for 63 months, and another patient (Case No. 6) maintained stable disease for 18 months after CKRS. Two of the four patients diagnosed with PPTID showed complete regression during the follow-up period (140 months and 26 months, respectively), one patient showed a partial response for 58 months, and the remaining patient showed tumor progression at 14 months after CKRS. After progression was confirmed, the patient underwent craniotomy for tumor removal. Via an occipital-transtentorial approach, more than $90 \%$ of the tumor was removed. The histologic features were not changed significantly compared with initial results, except for an increase of Ki-67 index from 30\% to 50\%. Because tumor progression was confirmed on MRI two months after surgery, we tried a PCV (Procarbazine, Lomustine, and Vincristine) chemotherapy. However, the tumor was progressing very aggressively, increasing to $48^{*} 30 \mathrm{~mm}$ at three months. Finally, the patient died at 21 months after CKRS and 7 months after craniotomy. All patients without recurrence were alive and there were no acute or late complications related to CKRS. A detailed clinical summary of these patients is shown in table 1.

Table 1. Clinical summary of six cases with pineal parenchymal tumor treated with cyberknife radiosurgery.

\begin{tabular}{|c|c|c|c|c|c|c|c|c|c|c|c|c|c|c|c|c|}
\hline Cases & Sex & Age & $\begin{array}{c}\text { Initial } \\
\text { symptoms }\end{array}$ & T1 & $\mathrm{T} 2$ & $\begin{array}{c}\mathrm{T1} \\
\text { enhancement }\end{array}$ & Surgery & Pathology & $\left|\begin{array}{l}\mathrm{Ki}- \\
67\end{array}\right|$ & $\begin{array}{l}\text { Marginal } \\
\text { Dose (Gy) }\end{array}$ & $\begin{array}{l}\text { Isodose } \\
\text { Curve (\%) }\end{array}$ & Fraction & \begin{tabular}{|c|} 
Planned \\
Tumor \\
volume (cc)
\end{tabular} & $\begin{array}{c}\text { Follow-up } \\
\text { period }\end{array}$ & esponses: & Survival \\
\hline 1 & M & 8 & Headache & iso & $\begin{array}{l}\text { iso to } \\
\text { high }\end{array}$ & enhancing & \begin{tabular}{|c|} 
ETV \\
+ biopsy
\end{tabular} & PPTID & 3 & 18 & 85 & 3 & 2.1 & 140 & $C R$ & Yes \\
\hline 2 & M & 33 & Diplopia & $\begin{array}{l}\text { iso to } \\
\text { low }\end{array}$ & iso & enhancing & \begin{tabular}{|c|} 
ETV \\
+ biopsy \\
\end{tabular} & PPTID & 30 & 18 & 80 & 3 & 3.8 & 22 & PD & No \\
\hline 3 & $\mathrm{~F}$ & 46 & Headache & low & $\begin{array}{c}\text { iso to } \\
\text { high }\end{array}$ & enhancing & \begin{tabular}{|c|} 
ETV \\
+ biopsy
\end{tabular} & PC & 4 & 18 & 80 & 3 & 1.8 & 63 & $P R$ & Yes \\
\hline 4 & $\mathrm{~F}$ & 58 & $\begin{array}{c}\text { Memory } \\
\text { impairment }\end{array}$ & low & high & enhancing & \begin{tabular}{|c|}
$\begin{array}{c}\text { ETV } \\
+ \text { biopsy }\end{array}$ \\
\end{tabular} & PPTID & 5 & 18 & 80 & 3 & 1.4 & 58 & $P R$ & Yes \\
\hline 5 & $\mathrm{~F}$ & 56 & $\begin{array}{c}\text { Gait } \\
\text { disturbance }\end{array}$ & low & high & enhancing & 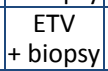 & PPTID & 15 & 18 & 80 & 3 & 1.8 & 26 & $C R$ & Yes \\
\hline 6 & $\mathrm{~F}$ & 48 & Headache & $\begin{array}{l}\text { iso to } \\
\text { low }\end{array}$ & $\begin{array}{l}\text { iso to } \\
\text { high }\end{array}$ & enhancing & \begin{tabular}{|c|} 
ETV \\
+ biopsy \\
\end{tabular} & PC & 1 & 18 & 80 & 3 & 7.4 & 18 & SD & Yes \\
\hline
\end{tabular}

\section{Illustrative Cases}

\section{Case 1 (Case No. 1)}

An 8-year-old male patient with mental retardation was referred for hydrocephalus and a pineal region tumor found on computed tomography performed due to minor head trauma. MRI revealed a $2.0 \times 1.6 \times 1.4 \mathrm{~cm}$ highly enhancing mass in the pineal region with obstructive hydrocephalus (figure 1a). We performed ETV and tumor biopsy, and histologic diagnosis was PPTID. After two weeks, he underwent fractionated CKRS with a marginal dose of 18 Gy as the primary treatment modality. After six months, MRI showed a marked decreased in the size of the tumor. Finally, the mass disappeared completely after two years of CKRS (figure 1b). No evidence of recurrence was confirmed at the last follow-up visit (140 months).

\section{Case 2 (Case No. 3)}

A 46-year-old female patient presented with a severe headache for three months. MRI showed a $1.3 \times 1.7 \times 1.4 \mathrm{~cm}$ lobulated and highly enhancing mass in the pineal region with obstructive hydrocephalus (figure 2a). We performed ETV and tumor biopsy, and the histologic diagnosis was pineocytoma. After one month, she underwent fractionated CKRS with a marginal dose of 18 Gy as the primary treatment modality. After two years, MRI showed a more than $50 \%$ decrease in tumor size (figure 2b), which was maintained for 63 months. 


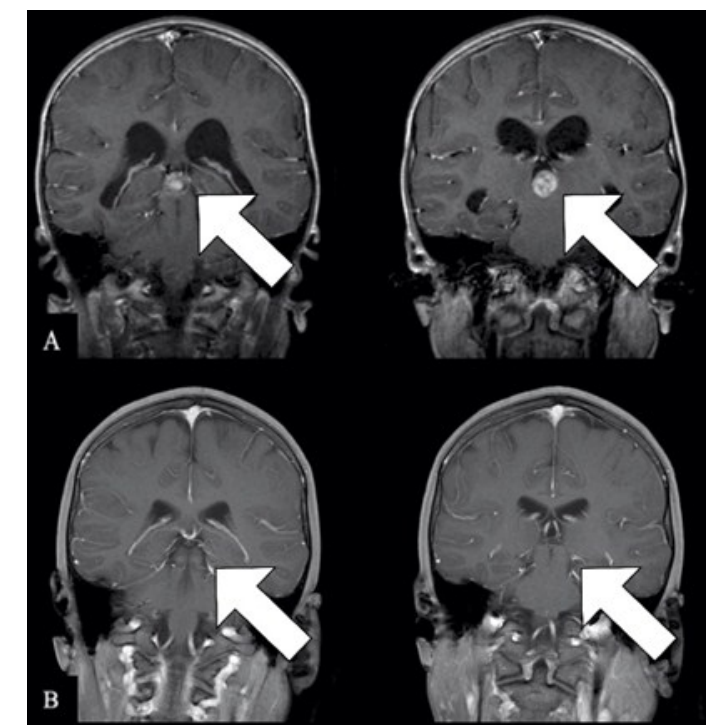

Figure 1. Radiologic findings of an 8-year-old male patient diagnosed with PPTID. a) Initial MRI showed a highly enhancing pineal lesion. b) Two years after CKRS, the mass had completely resolved.

\section{DISCUSSION}

Pineocytoma accounts for about $20 \%$ of all PPTs and typically occurs in adult patients, with a mean age of 43 years at diagnosis( ${ }^{(1)}$. It is a well -demarcated and exclusively localized solid mass without infiltration in the pineal region. The reported five-year survival rate ranges from $86 \%$ to $91 \%(1,3)$. Extent of surgery is considered a major prognostic factor in patients with pineocytoma (10). Gross total resection seems reasonable, but aggressive surgery should be carefully considered given that complete tumor resection is only achieved in half of patients, and perioperative mortality and morbidity case occurs in a few cases. SRS has been suggested as an alternative treatment modality for primary, adjuvant, or recurrent cases. Recent studies showed a greater than $80 \%$ local tumor control rate for five years and a median progressionfree survival ranging from 17.3 to 100 months (10-13,17-21). In our study, both patients with PC presented with symptoms of hydrocephalus and underwent SRS as the primary therapy after ETV and biopsy. Both patients had stable disease at the last follow-up visit at a median 40.5 (range 18-63) months. These findings suggest that SRS can be effective as the primary

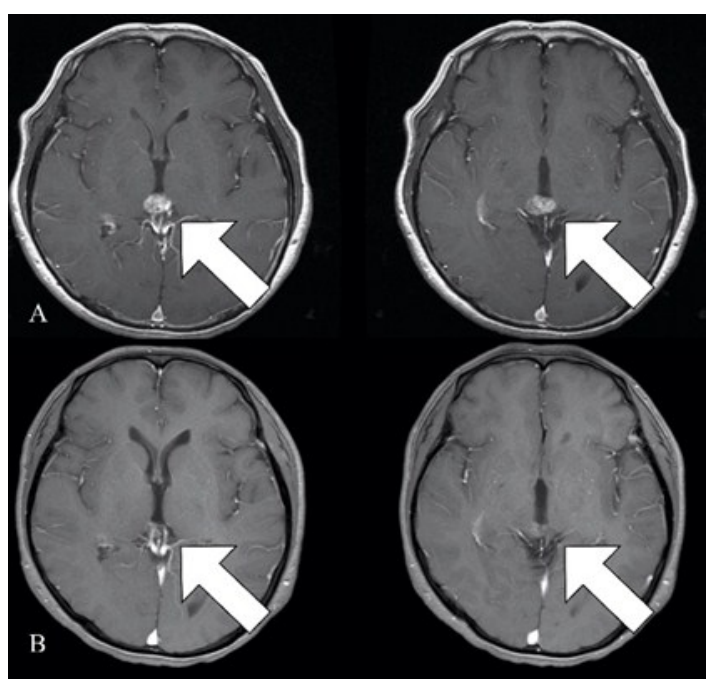

Figure 2. Radiologic findings of a 46-year-old female patient diagnosed with PC. a) The initial MRI showed a highly enhancing pineal lesion. b) Two years after CKRS, the mass had decreased in size.

treatment modality for local tumor control in patients with pineocytoma.

PPTID accounts for about $45 \%$ of all PPT and can occur in patients of any age, although it typically occurs in adult patients, with a mean age of 41 (range 1-83) years at diagnosis $(1,3)$. In contrast with pineocytoma, PPTID has variable biologic and clinical features. Five-year survival rates were 39 to $74 \%$ according to histological features (1,3-5). Number of mitoses per 10 high-power fields, anti NFP IHC, and ki-67 proliferation index are considered prognostic factor $(4,5,13,14,22)$. Due to the rarity and heterogeneity of this tumor, the optimal treatment of PPTID among surgical resection, radiotherapy, SRS, and chemotherapy is more unclear than for other PPT. SRS has been attempted in patients with PPTID in recent studies and showed a $50 \%$ progression-free rate, $100 \%$ LTC at 24 months, and $100 \%$ LTC at 77.6 months ${ }^{(11-13)}$. In our study, three of the four patients with PPTID showed no local recurrence over a median 49.8 (range 26-111) months, but one patient showed progression 22 months after SRS. Notably, this patient had a $30 \% \mathrm{Ki}$ index, while the other patients had a mean index of $7.6 \%$ (range 3-15). It seems that lower-risk groups with a low Ki index can be treated with 
RSR, but the optimal therapeutic strategy should be carefully considered based on histologic features in higher-risk groups with a high $\mathrm{Ki}$ index, like patient 2 in our study.

This study has several limitations, including a small number of cases examined in a retrospective manner and lack of a comparison group. Further studies are needed to elucidate the efficacy and safety of SRS as a primary modality for treatment of PPT.

\section{CONCLUSIONS}

Our strategy consisted of CKRS as the primary therapy after ETV and tumor biopsy for patients with PPT (except pineoblastoma). Our findings indicate this approach as effective and safe, but more careful consideration is needed for higher-risk patients with PPTID.

\section{Conflicts of interest: Declared none.}

\section{REFERENCES}

1. Louis DN, Ohgaki H, Wiestler $O D$, Cavenee WK, Burger PC, Jouvet $A$, et al. (2007) The 2007 WHO classification of tumours of the central nervous system. Acta neuropathologica, 114(2): 97-109.

2. Fauchon $F$, Jouvet $A$, Paquis $P$, Saint-Pierre $G$, Mottolese $C$, Hassel MB, et al. (2000) Parenchymal pineal tumors: a clinicopathological study of 76 cases. International Journal of Radiation Oncology* Biology* Physics, 46(4): 959-68.

3. Louis $D N$, Perry $A$, Reifenberger $G$, Von Deimling A, Figarella-Branger D, Cavenee WK, et al. (2016) The 2016 WHO classification of tumors of the central nervous system: $a$ summary. Acta neuropathologica, 131(6): 803-20.

4. Choque-Velasquez J, Resendiz-Nieves JC, Jahromi BR, Colasanti $R$, Raj R, Tynninen O, et al. (2019) Pineal Parenchymal Tumors of Intermediate Differentiation: A long-Term Follow-Up Study in Helsinki Neurosurgery. World neurosurgery, 122: e729-e39.

5. Mallick S, Benson R, Rath $G$ (2016) Patterns of care and survival outcomes in patients with pineal parenchymal tumor of intermediate differentiation: An individual patient data analysis. Radiotherapy and Oncology, 121(2): 204-8.

6. Fauchon $F$, Hasselblatt $M$, Jouvet $A$, Champier J, Popovic $M$, Kirollos $R$, et al. (2013) Role of surgery, radiotherapy and chemotherapy in papillary tumors of the pineal region: a multicenter study. Journal of neuro-oncology, 112(2): 223 -31 .

7. Amendola BE, Wolf A, Coy SR, Amendola MA, Eber D (2005) Pineal tumors: analysis of treatment results in 20 patients. Journal of neurosurgery, 102(Special_Supplement): 175-9.

8. Bruce JN and Ogden AT (2004) Surgical strategies for treating patients with pineal region tumors. Journal of neuro-oncology, 69(1-3): 221-36.

9. Konovalov AN and Pitskhelauri DI (2003) Principles of treatment of the pineal region tumors. Surgical neurology, 59 (4): $252-70$.

10. Wilson DA, Awad A-W, Brachman D, Coons SW, McBride H, Youssef $E$, et al. (2012) Long-term radiosurgical control of subtotally resected adult pineocytomas. Journal of neurosurgery, 117(2): 212-7.

11. Park JH, Kim JH, Do Hoon Kwon CJK, Khang SK, Cho YH (2015) Upfront stereotactic radiosurgery for pineal parenchymal tumors in adults. Journal of Korean Neurosurgical Society, 58(4): 334.

12. Balossier A, Blond S, Touzet $G$, Sarrazin T, Lartigau E, Reyns $N$ (2015) Role of radiosurgery in the management of pineal region tumours: indications, method, outcome. Neurochirurgie, 61(2-3): 216-22.

13. Iorio-Morin $C$, Kano $H$, Huang $M$, Lunsford $L D$, Simonová $G$, Liscak $R$, et al. (2017) Histology-stratified tumor control and patient survival after stereotactic radiosurgery for pineal region tumors: A report from the international gamma knife research foundation. World neurosurgery, 107: 974-82.

14. Yu T, Sun X, Wang J, Ren X, Lin N, Lin S (2016) Twentyseven cases of pineal parenchymal tumours of intermediate differentiation: mitotic count, Ki-67 labelling index and extent of resection predict prognosis. Journal of Neurology, Neurosurgery \& Psychiatry, 87(4): 386-95.

15. Li W, Zhang B, Kang W, Dong B, Ma X, Song J, et al. (2015) Gamma knife radiosurgery (GKRS) for pineal region tumors: a study of 147 cases. World journal of surgical oncology, 13 (1): 304.

16. Benedict $S H$, Yenice KM, Followill D, Galvin JM, Hinson W, Kavanagh B, et al. (2010) Stereotactic body radiation therapy: the report of AAPM Task Group 101. Medical physics, 37(8): 4078-101.

17. Reyns $N$, Hayashi $M$, Chinot $O$, Manera L, Péragut J-C, Blond S, et al. (2006)The role of Gamma Knife radiosurgery in the treatment of pineal parenchymal tumours. Acta neurochirurgica, 148(1): 5-11.

18. Lekovic GP, Gonzalez LF, Shetter AG, Porter RW, Smith KA, Brachman D, et al. 2007) Role of Gamma Knife surgery in the management of pineal region tumors. Neurosurgical focus, (23(6): E11.

19. Mori Y, Kobayashi T, Hasegawa T, Yoshida K, Kida Y (2009) Stereotactic radiosurgery for pineal and related tumors. Progress in neurological surgery, 23: 106-18.

20. Kano $H$, Niranjan A, Kondziolka D, Flickinger JC, Lunsford D (2009) Role of stereotactic radiosurgery in the management of pineal parenchymal tumors. Progress in neurological surgery, 23: 44-58. 
21. Yianni J, Rowe J, Khandanpour N, Nagy G, Hoggard N, Radatz $M$, et al. (2012) Stereotactic radiosurgery for pineal tumours. British journal of neurosurgery, 26(3): 361-6.
22. Amato-Watkins AC, Lammie A, Hayhurst C, Leach P (2016) Pineal parenchymal tumours of intermediate differentiation-An evidence-based review of a new pathological entity. British journal of neurosurgery, 30(1): 11-5. 